University of Nebraska - Lincoln

DigitalCommons@University of Nebraska - Lincoln

2008

\title{
A call for longitudinal research in positive organizational behavior
}

James B. Avey

Central Washington University, aveyj@cwu.edu

Fred Luthans

University of Nebraska - Lincoln, fluthans1@unl.edu

Ketan $\mathrm{H}$. Mhatre

University of Nebraska - Lincoln

Follow this and additional works at: https://digitalcommons.unl.edu/leadershipfacpub

Part of the Management Sciences and Quantitative Methods Commons

Avey, James B.; Luthans, Fred; and Mhatre, Ketan H., "A call for longitudinal research in positive organizational behavior" (2008). Leadership Institute Faculty Publications. 7.

https://digitalcommons.unl.edu/leadershipfacpub/7

This Article is brought to you for free and open access by the Leadership Institute at DigitalCommons@University of Nebraska - Lincoln. It has been accepted for inclusion in Leadership Institute Faculty Publications by an authorized administrator of DigitalCommons@University of Nebraska - Lincoln. 
Published in Journal of Organizational Behavior 29:5 (2008), pp. 705-711; doi: 10.1002/job.517

Copyright (C 2008 John Wiley \& Sons, Ltd. Used by permission. http://www.interscience.wiley.com

Submitted October 19, 2007; accepted November 13, 2007.

“THE INCUBATOR”

\title{
A call for longitudinal research in positive organizational behavior
}

\author{
James B. Avey, ${ }^{1}$ Fred Luthans, ${ }^{2}$ and Ketan H. Mhatre ${ }^{2}$ \\ 1 Department of Management, Central Washington University \\ 2 Department of Management, Gallup Leadership Institute, University of Nebraska-Lincoln \\ Corresponding author - James B. Avey, Central Washington University, Management, \\ 400 E. University Way, Ellensburg, WA 98926, USA E-mail: aveyj@cwu.edu
}

\begin{abstract}
After defining and providing the current status of psychological capital (PsyCap), we address the need to better understand its stability (i.e., state $v$ s. trait). Specifically, we issue a call for longitudinal research. The balance of this "incubator" article examines the relevance, potential obstacles, and advantages of this design in meeting PsyCap's future research needs.
\end{abstract}

\section{Introduction}

Several years ago in this journal, positive organizational behavior (POB) was identified as a way to add needed focus and the field was charged to advance its understanding through basic research (Luthans, 2002; Wright, 2003). Reflecting on the current research needs of POB and its application through psychological capital or PsyCap (see Luthans, Youssef, \& Avolio, 2007), two closely related important issues immediately surface. First is the need for the better understanding of the so-called "state-trait continuum" so central to the meaning and application of POB and PsyCap. The second more specific need is for longitudinal research designs that advance knowledge of if and how PsyCap fluctuates over time and the corresponding effects on important organizational outcomes such as employee development and performance.

This incubator piece addresses these two issues by first briefly introducing the meaning and current status of PsyCap. Next, the key distinction between traits and states in PsyCap is discussed with an emphasis on a continuum perspective versus a dichotomy perspective. This background is then followed by a brief description of the advantages that longitudinal research designs can offer to the better understanding of this state-trait continuum. Finally, in the spirit of "incubation," some specific questions for future research that lend themselves to longitudinal designs conclude the article. 


\section{Positive Psychological Capital}

Drawn from positive psychology and POB, psychological capital or PsyCap is defined as "an individual's positive psychological state of development and is characterized by: (1) having confidence (efficacy) to take on and put in the necessary effort to succeed at challenging tasks; (2) making a positive attribution (optimism) about succeeding now and in the future; (3) persevering toward goals and, when necessary, redirecting paths to goals (hope) in order to succeed; and (4) when beset by problems and adversity, sustaining and bouncing back and even beyond (resilience) to attain success" (Luthans, Youssef et al., 2007, p. 3). Although other positive constructs such as wisdom, well being, emotional intelligence, courage, and even spirituality have been considered as potential PsyCap (e.g., see Chapters 6 and 7 in Luthans, Youssef et al., 2007), efficacy, optimism, hope, and resilience have been featured as best meeting the defined PsyCap inclusion criteria of being state-like and demonstrating performance impact.

Following the above definition, PsyCap theory development indicates that the four dimensions possess a common underlying positive agentic capacity, that is, those with high PsyCap tend to be more determined, expend more effort, expect success, maneuver obstacles more effectively, and bounce back from setbacks more readily (Luthans, Avolio, Avey \& Norman, 2007; Luthans, Youssef et al., 2007). Besides conceptual support, there is also empirical evidence that the four constructs when combined into PsyCap form a core factor. Using a competing measurement models analysis, PsyCap has been found to be best measured as a second-order factor, whereas each dimension (efficacy, hope, optimism, and resilience) loads onto an overall core factor (PsyCap) (Luthans, Avolio et al., 2007).

As to performance impact, empirical research across multiple samples indicates that employees' overall PsyCap relates better to performance and satisfaction than each of the four factors individually, and there is a strong positive relationship between PsyCap and performance (both manager rated and objectively measured) and satisfaction (Luthans, Avolio et al., 2007). The critical differentiator of this defined PsyCap from most other positive approaches, however, is the state-like, open to development criterion. To date, there is both conceptual (Luthans, Avey, Avolio, Norman, \& Combs, 2006; Luthans, Youssef et al., 2007) and beginning empirical evidence (Luthans, Avey, \& Patera, 2008) that PsyCap can be developed in short training interventions. Nevertheless, the major challenge facing PsyCap remains this state-trait distinction. After first providing a brief background of the issues, we propose that longitudinal research designs may contribute to meeting this challenge of the better understanding and effective application of PsyCap.

\section{State or Trait? A Continuum Perspective}

Despite the growing evidence that PsyCap has performance impact, no research to date has applied a longitudinal research design to test the stability of this core construct. If the assumption is that a positive construct is a stable trait, this tempers the need to study it longitudinally because the variance in measurement would potentially be less robust in nature and, as a consequence, less likely to be predictive. However, if PsyCap is defined as state-like (Luthans, 2002; Luthans, Avolio et al., 2007; Luthans, Youssef, et al., 2007), then there is a heightened need for longitudinal research designs.

Much of the confusion surrounding the stability issue in POB and PsyCap is that each of the four constructs that make up PsyCap have been presented in the positive psychology literature as both a state and a trait. For example, there are widely recognized trait and state theories and measures for 
each of the four constructs. Snyder has both trait hope and state hope scales and although optimism has traditionally been portrayed as a trait, Seligman's concept of "learned optimism" indicates that it is state-like and open to development. The same is true of efficacy (i.e., specific or general efficacy) and resiliency. Such seemingly contradictory conceptualizations in reference to PsyCap need to be settled empirically (i.e., through longitudinal designs).

Many years ago, Allport established that traits are stable across time and contexts. Despite this agreement in classic psychology of the nature of traits (and states), when applied to the study of POB, Wright (2007, p. 180) has noted "there is a dire need for the positive movements to reach a conceptual consensus regarding exactly what temporal period constitutes a state and what constitutes a trait." Given the emphasis on stability of traits and fluctuation of states, it follows that an understanding of construct stability estimates over time may yield important information to understanding the state-trait distinction that is so vital to not only the research, but also for PsyCap application (e.g., for selection or development).

In a relevant meta-analysis, Conley (1984) conducted test-retest reliability statistics over several years for intelligence, personality, and self-opinion constructs. It was found that intelligence was more stable than personality which was more stable than self-opinions. Given that a clear dichotomy did not emerge, this study provided preliminary evidence that states and traits may be best understood along a continuum. Using Conley's study as a point of departure, we recently conducted a short longitudinal test (3 time points over four weeks) of PsyCap, personality (conscientiousness), core self-evaluation traits and positive emotions in order to better understand the relative stability of PsyCap (Luthans, Avolio et al., 2007). After disattenuating for internal reliability, the corrected results indicated support for this continuum perspective of states and traits. Specifically, this preliminary research found core self-evaluation traits were the most stable (.87), followed by personality (.76), PsyCap (.52), and positive emotions (.46). Therefore, it follows that perhaps the optimal method of considering states and traits in the domain of POB is the degree of stability, rather than the potentially limiting dichotomous thinking of a stable trait versus an unstable state.

\section{The Relevance of Longitudinal Designs}

One value of a continuum perspective of traits and states is that POB researchers may be more likely to include individual factors in longitudinal research that were previously dismissed as "stable" $^{\prime \prime}$. For example, if researchers were to utilize a longitudinal research design to better understand the relationship between PsyCap and performance and assumed PsyCap to be stable, they may measure PsyCap at time 1, and then track performance thereafter. The assumption of stability of PsyCap (or any other positive variable) rules out significant within person variance which can be critical to advancing knowledge and has implications for human resource development. Contrarily, if researchers expect significant within person variance in PsyCap scores over time (i.e., an unstable assumption), then they may measure PsyCap at each time point to test for evidence of concepts such as feedback loops or reciprocal causation.

The acknowledgement of variance over time in PsyCap is critical to the application of longitudinal research designs which have been largely absent in this specific PsyCap area of study and most other areas of POB. There are several ways we see where the application of longitudinal research designs can extend $\mathrm{POB}$ understanding and application. However, before discussing these advantages of longitudinal data, it is first necessary to briefly mention why the field of POB has not conducted longitudinal research. 


\section{Overcoming the Obstacles in Longitudinal Research}

Following Ployhart, Holtz and Bliese (2002, p. 456) who note "perceived difficulty in collecting and analyzing longitudinal data are probably among the most important factors prohibiting longitudinal leadership research," we propose that one reason there is so little longitudinal research is the perceived disruptive interference in the organizations studied. Unlike psychology, in POB research the context of the organization is of primary importance. For example, gaining access to employees, obtaining permission to survey and/or observe them, and then gathering often sensitive, private information such as on performance indices, can be a challenge. While gaining initial access to a research site may be difficult in itself, requesting permission for repeated measures in a longitudinal design raises the practical question from the managerial staff of "why ask the same thing over and over."

One way to address such problems and practical concerns with repeated measures is keeping the survey questions to a minimum. For example, we recently have reduced the 24-item PsyCap questionnaire (PCQ) (see Luthans, Avolio et al., 2007; Luthans, Youssef et al., 2007) down to 12 items. This PCQ-12 has been demonstrated in a number of samples to have acceptable reliability and support for construct validity. We recommend as long as the psychometric criteria are met, as in the PCQ-12, shorter measures are more compatible for use in longitudinal research.

Another reason for the lack of longitudinal research designs in POB in general and PsyCap in particular is unfamiliarity with some of the analysis techniques. For example, drawing on regression models with which regression and general linear modeling researchers are comfortable, Ployhart and colleagues (2002) present the longitudinal data analysis technique called random coefficient modeling (RCM). This RCM is based on regression and allows for many of the challenges encountered in longitudinal research such as correlated responses and high subject mortality rates. In sum, an appreciation of the potential disruption in organizations from repeated measure designs, as well as researchers utilizing techniques such as RCM may assist in answering the call for more longitudinal research in POB and PsyCap.

\section{Advantages of Longitudinal Designs}

In an emerging field such as POB and PsyCap, where so many research questions have yet to be addressed, longitudinal designs may provide insights otherwise undetectable. For example, as highlighted by Wright (2007), one advantage of longitudinal research is that it allows the researcher to model time as an independent variable. Whereas theories often explicitly state the importance of time (e.g., employee socialization or leadership development), longitudinal data actually allow the use of time as a research variable. Second, as previously highlighted in Conley's (1984) meta-analysis, longitudinal research provides stability estimates of constructs allowing researchers the opportunity to distinguish those constructs in which they may expect more (or less) within person variance over time. Third is the importance of between person variance. Typical cross sectional research seeks between person variance; that is, those higher in variable $X$ (let us say PsyCap) will be higher in variable $Y$ (let us say psychological well-being or PWB), whereas those lower in PsyCap will be lower in PWB. Longitudinal data also have the advantage of providing within person variance which is the change in individuals over time. Longitudinal data can address questions such as the fluctuation in scores (do individuals significantly differ in their responses over time) or questions involving both within and between person variance such as do neurotics have greater within person change (e.g., fluctuation in responses) in attitude measures than those who are emotionally stable. 
A fourth type of information that longitudinal research can yield is an examination of the order of change in response patterns. For example, if PsyCap increases and this is followed by an increase in PWB; and next PsyCap decreases followed by a decrease in PWB, there is stronger inference of causality than simply a cross-sectional correlation. While there may be no substitute for a true experimental design in terms of inferring causality, a longitudinal design can be helpful when trying to tease apart variables with complex relationships with questions such as, does cognition precede emotion, vice versa, or both? The ability to study such patterns over time is likely to illuminate issues otherwise not detected.

A fifth type of information which longitudinal research can yield is the monitoring of interventions to determine length of effects. For example, the beginning PsyCap training intervention research does not track responses over time (e.g., Luthans et al., 2008). By using a longitudinal design to include multiple post-tests, researchers can identify the extent to which the intervention effects last and if there is a delayed effect where there is no significant change at time 2, but it does emerge at time 3 .

At this point in presenting the advantages of longitudinal designs, it seems important to reiterate that "pragmatism" often inappropriately dictates when variable measures are taken (Wright, 1997, p. 202). If, for example, time 1 of a training intervention pre-test for PsyCap development was taken immediately following a performance evaluation by a supervisor, the data may be confounded. Therefore, data collections in these longitudinal studies (such as those monitoring training results) must include appropriate and intentional times of data collection, and perhaps even include the role of time as an independent variable (Wright, 1997). In addition to monitoring training results, the longitudinal design could also identify which types of training participants are likely to experience longer term sustainable development rather than short-term changes.

In summary, there are multiple advantages to longitudinal research over the traditional cross sectional and even experimental research designs. Although such designs have been advocated through the years in the overall field of organizational behavior, the patterns of rich data gained from longitudinal research seem especially valuable to advancing POB and PsyCap.

\section{Future Research}

Given the advantages of longitudinal research designs noted above and the types of questions these designs can answer, we suggest that there is an untapped opportunity to advance the understanding of POB/PsyCap and its effective application. In particular, we suggest there are several areas for future research in POB and PsyCap that would seem to especially benefit from longitudinal designs.

First, advances in the understanding of a state versus trait continuum must be obtained through longitudinal research. The positive approach in general includes both psychological states and traits. A richer understanding of the stability of all positive constructs would enable both scholars and practitioners to determine which capacities are indeed open to development and which are more suitable for selection.

Second, longitudinal research can be applied to test hypotheses on the length of intervention effects. For example, our preliminary research has found that positive PsyCap can be significantly developed through a short web-based training intervention (Luthans et al., 2008) and our work both face-to-face and on-line with a number of different samples using 1-3 hours training interventions is yielding similar results. A meaningful extension of this work would be a comparison of various types of intervention mediums (e.g., face-to-face vs. web-based) or length (e.g., 2 hours vs. 2 weeks). Theory-driven hypotheses on the length of intervention effects could also be tested longitudinally. 
A third use of longitudinal designs in future research could be testing theory-driven hypotheses on the extent to which $\mathrm{POB}$ constructs are attributable to the person and how much is attributable to the context. However, rather than a simple study on within and between person variance, hypotheses could be derived and tested on the role of contextual factors such as group dynamics, organizational culture, leadership influence, demographic group impact, and job design that influence POB constructs (such as PsyCap or PWB) over time.

Still another future research area in POB and PsyCap that may benefit from longitudinal designs could be the comparison of variation patterns in various positive states in stable contexts and in contexts that typify danger or other types of extremis situations such as high reliability organizations (e.g., nuclear power plants or healthcare facilities) or military combat. For example, it may be plausible to assert that leaders in a hospital emergency room or combat (or police or fire) teams may experience more robust positive construct patterns over time (e.g., higher highs and lower lows) with changes in the environment, than those in less extremis contexts.

\section{Conclusion}

In conclusion, the purpose of this incubator is to highlight several issues to advancing research in $\mathrm{POB}$ in general and psychological capital in particular. After introducing the positive focus to organizational behavior in JOB several years ago (see Luthans, 2002; Wright, 2003), the time seems right to consider and incubate some needed research methodological issues that have surfaced for future better understanding and effective application of POB and PsyCap. In particular, longitudinal research designs seem especially needed at this juncture of the POB field of study. Not only are there specific types of information that only longitudinal data can yield, but there are also several important opportunities to apply this type of research that will help attain one of the overall goals of POB, that is, understand and enhance what is right with people and what is working in organizations. Overall, the study and application of POB has shown considerable growth of understanding and application in recent years and hopefully this discussion will stimulate and incubate longitudinal research for the sustainable growth of this important, positive field of study and application.

\section{About the Authors}

James B. Avey is Assistant Professor of Management at Central Washington University. He earned his Ph.D. from the Gallup Leadership Institute at the University of Nebraska-Lincoln; his research interests include positive psychological capital, psychological ownership, and authentic leadership.

Fred Luthans is the George Holmes University Distinguished Professor of Management in the Department of Management and Gallup Leadership Institute, University of NebraskaLincoln. He is a past president of the Academy of Management and the editor of three journals; his current research revolves around $\mathrm{POB}$ and psychological capital.

Ketan H. Mhatre is a doctoral candidate at the Gallup Leadership Institute in the University of Nebraska-Lincoln with an expected graduation in 2008. His research interests include authentic leadership development, influence processes, and impression management. 


\section{References}

Conley, J. J. (1984). The hierarchy of consistency: A review and model of longitudinal findings on adult individual differences in intelligence, personality, and self-opinion. Personality and Individual Differences, 5, 11-25.

Luthans, F. (2002). The need for and meaning of positive organizational behavior. Journal of Organizational Behavior, 23, 695-706.

Luthans, F., Avey, J. B., Avolio, B. J., Norman, S. M., \& Combs, G. M. (2006). Psychological capital development: Toward a micro-intervention. Journal of Organizational Behavior, 27, 387-393.

Luthans, F., Avolio, B. J., Avey, J. B., \& Norman, S. M. (2007). Psychological capital: Measurement and relationship with performance and satisfaction. Personnel Psychology, 60, 541-572.

Luthans, F., Youssef, C. M., \& Avolio, B. J. (2007). Psychological Capital. New York: Oxford University Press.

Luthans, F., Avey, J. B., \& Patera, J. L. (2008). Experimental analysis of a web-based training intervention to develop positive psychological capital. Academy of Management Learning and Education, (in press).

Ployhart, R. E., Holtz, B. C.,\& Bliese, P. D. (2002). Longitudinal data analysis: Applications of random coefficient modeling to leadership research. The Leadership Quarterly, 13, 455-486.

Wright, T. A. (1997). Time revisited in organizational behavior. Journal of Organizational Behavior, 18, 201-204.

Wright, T. A. (2003). Positive organizational behavior: An idea whose time has truly come. Journal of Organizational Behavior, 24, 437-442.

Wright, T. A. (2007). A look at two methodological challenges for scholars interested in positive organizational behavior. In D. Nelson, \& C. L. Cooper (Eds.), Positive organizational behavior: Accentuating the positive at work (pp. 177-190). Thousand Oaks, CA: Sage. 